\title{
Effect of Sympatholytic Therapy on Circadian Cardiac Autonomic Activity in Non-Diabetic Chronic Kidney Disease
}

\author{
Hisaki Makimoto, ${ }^{1,2 *}$ MD, Kohei Shimizu, ${ }^{3, *}$ MD, Katsuhito Fujiu, ${ }^{1,4}$ MD, Tina Lin, ${ }^{5}$ MD, \\ Tsukasa Oshima, ${ }^{1}$ MD, Eisuke Amiya, ${ }^{1}$ MD, Kenichiro Yamagata, ${ }^{1}$ MD, Toshiya Kojima, ${ }^{1}$ MD, \\ Masao Daimon, ${ }^{1} \mathrm{MD}$, Ritsuko Nagatomo, ${ }^{4} \mathrm{MD}$, Kayo Waki, ${ }^{4} \mathrm{MD}$, \\ Christian Meyer, ${ }^{2} \mathrm{MD}$ and Issei Komuro, ${ }^{1} \mathrm{MD}$
}

\begin{abstract}
Summary
Although beta-blockade itself is not a first choice for chronic kidney disease (CKD) patients, alpha-betablockers (ABB) do improve their prognoses. This study's aim was to evaluate the effect of beta-selectiveblockers (BSB) and $\mathrm{ABB}$ on circadian cardiac autonomic activity in CKD patients.

The study consisted of 496 non-diabetic individuals who underwent 24-hour Holter monitoring (149 CKD patients and 347 controls without CKD). Using heart rate variability analysis, we evaluated the proportion of NN50 and the high-frequency component (reflecting parasympathetic activity), and low- to high-frequency ratio (reflecting sympathovagal balance). These indices were evaluated by regression analysis incorporating gender, age, related comorbidities, and medications. BSB increased vagal activity only in the day-time and not the night-time in controls. In CKD patients, BSB was significantly related to higher vagal activity throughout the day and with lower sympathovagal balance at night. The night sympathovagal balance of CKD patients taking $\mathrm{ABB}$ was significantly higher than that of CKD patients taking BSB, which was the only significant difference between the effects of BSB and ABB.

The sympatholytic therapy effect is different depending on CKD presence and whether patients are treated with $\mathrm{BSB}$ or $\mathrm{ABB}$. In $\mathrm{CKD}$ patients without severe heart failure, BSB could be associated with higher parasympathetic activity and lower sympathovagal balance compared to ABB.
\end{abstract}

(Int Heart J 2018; 59: 1352-1358)

Key words: Cardiac autonomic function, Kidney dysfunction, Heart rate variability, $\beta$-blocker

$\mathrm{H}$ eart failure patients with higher sympathetic activity experience higher mortality. ${ }^{1,2)}$ Treatment with $\beta$-blockers improves these patients' outcomes. ${ }^{3)}$ The current guidelines recommend the use of $\beta$ blockade as a class IA indication. ${ }^{4)}$

Patients with higher sympathetic activity and chronic kidney disease (CKD) also experience higher mortality. ${ }^{5)}$ However, in contrast to heart failure patients, $\beta$-blockers are not recommended as first line therapy for CKD patients. $\left.{ }^{6}\right)$ Instead, clinicians favor renin-angiotensin system blockers; in fact, previous studies demonstrated that Atenolol is not as effective at slowing renal disease progression compared to Lisinopril and calcium channel blockers. ${ }^{7)}$

Recently we reported higher cardiac sympathetic activity and impaired parasympathetic activity in CKD patients. ${ }^{8)}$ Based on these results, lowering sympathetic ac- tivity in $\mathrm{CKD}$ patients may result in a prognostic improvement. Wali, et al. reported that $\alpha \beta$-blockers (ABB) improved outcomes in heart failure patients with CKD. ${ }^{9)}$

$\beta$-blockers decrease all-cause and cardiovascular mortality in CKD patients who have heart failure with reduced left ventricular ejection fraction (LVEF). ${ }^{10)}$ However, it has not yet been clarified whether the use of $\beta$ blockers would benefit CKD patients without heart failure.

In this study, we compared the sympatholytic effect of $\beta$-blocking agents in CKD patients without heart failure to non-CKD individuals using heart rate variability (HRV) analysis. We also evaluated the difference between the effects of $\beta$-selective $\beta$-blockers (BSB) and $\mathrm{ABB}$ on cardiac autonomic function.

From the ${ }^{1}$ Department of Cardiovascular Medicine, The University of Tokyo Hospital, Tokyo, Japan, ${ }^{2}$ Department of Cardiology, Pulmonology and Vascular Medicine, University Hospital Duesseldorf, Dusseldorf, Germany, ${ }^{3}$ Department of Clinical Laboratory, The University of Tokyo Hospital, Tokyo, Japan, ${ }^{4}$ Department of Ubiquitous Health Informatics, Graduate School of Medicine, The University of Tokyo, Tokyo, Japan and ${ }^{5}$ Heart Care Victoria, Victoria, Australia.

* These authors contributed equally to this work.

Address for correspondence: Hisaki Makimoto, MD, Department of Cardiovascular Medicine, The University of Tokyo Hospital, 7-3-1, Hongo, Bunkyo-ku, Tokyo 113-8655, Japan. E-mail: h1sak1mak1m0t0@gmail.com

Received for publication September 30, 2017. Revised and accepted January 18, 2018

Released in advance online on J-STAGE October 25, 2018.

doi: 10.1536/ihj.17-561

All rights reserved by the International Heart Journal Association. 
Table I. Patients Characteristics

\begin{tabular}{lccc}
\hline & Group-NKD & Group-KD & P value \\
& $n=347$ & $n=149$ & \\
\hline Male & $150(43 \%)$ & $80(54 \%)$ & 0.032 \\
Age (years) & $58.6 \pm 15.4$ & $70.6 \pm 10.8$ & $<0.0001$ \\
Hypertension & $133(38 \%)$ & $97(65 \%)$ & $<0.0001$ \\
$\quad$ sBP (mmHg) & $126 \pm 18$ & $127 \pm 18$ & 0.73 \\
$\quad$ dBP (mmHg) & $75 \pm 12$ & $70 \pm 11$ & $<0.0001$ \\
Dyslipidemia & $99(29 \%)$ & $59(40 \%)$ & 0.016 \\
Hyperuremia & $16(5 \%)$ & $44(30 \%)$ & $<0.0001$ \\
Ischemic heart disease & $23(7 \%)$ & $24(16 \%)$ & 0.0010 \\
LVEF (\%) & $70 \pm 8$ & $69 \pm 10$ & 0.55 \\
LV Mass (g) & $143.0 \pm 45.1$ & $164.6 \pm 53.6$ & $<0.0001$ \\
Hemoglobin (g/dL) & $13.4 \pm 1.6$ & $12.5 \pm 1.9$ & $<0.0001$ \\
Serum creatinine (mg/dL) & $0.69 \pm 0.15$ & $1.72 \pm 1.85$ & $<0.0001$ \\
eGFR & $80.5 \pm 17.8$ & $41.2 \pm 15.4$ & $<0.0001$ \\
ACEi/ARB & $83(24 \%)$ & $80(54 \%)$ & $<0.0001$ \\
$\beta$-selective-blocking agent & $24(7 \%)$ & $26(17 \%)$ & 0.0004 \\
$\alpha \beta$-blocking agent & $19(5 \%)$ & $15(10 \%)$ & 0.064 \\
Ca antagonist & $70(20 \%)$ & $77(52 \%)$ & $<0.0001$ \\
\hline Chronic kidney disease patients & (2) & &
\end{tabular}

Chronic kidney disease patients were older and had significantly more comorbidities and drug therapies (see text for details).

\section{Methods}

Study population: The study population consisted of consecutive patients who underwent 24-hour holter ECGs between 2009 and 2011. The study was approved by the local Institutional Review Board. Patients meeting the following criteria were excluded from the study:

- Over 2,000 presystolic atrial or ventricular complex (PAC or PVC, respectively) beats per day Over 3,000 $\mathrm{PAC}$ and PVC per day

- Type 1 or 2 diabetes mellitus

- Congenital heart disease

- Atrial fibrillation

- Significant heart valve disease

- Hospital admission due to acute renal failure or congestive heart failure within the previous three months

- Chronic heart failure with New York Heart Association Functional Classification (NYHA) grade III or IV symptoms.

We enrolled 496 consecutive patients in this cohort study, assigning 149 patients with $<60 \mathrm{~mL} /$ minute/1.73 $\mathrm{m}^{2}$ to Group-KD and 347 individuals with $\geq 60 \mathrm{~mL} / \mathrm{min}$ ute $/ 1.73 \mathrm{~m}^{2}$ to Group-NKD. The number of patients with stage 3, 4, and 5 CKD were 120, 13, and 16 respectively. Patients' characteristics are shown in Table I.

Clinical data: Baseline clinical information was collected retrospectively based on clinical records. Ambulant blood pressure and hemoglobin $(\mathrm{Hb})$ were recorded. eGFR was determined based on the new Japanese coefficientmodified Modification of Diet in Renal Disease (MDRD) study equation. ${ }^{11)}$ Ischemic heart disease was defined as a history of over $75 \%$ stenosis in a coronary artery diagnosed on coronary angiography or coronary CT.

Echocardiography: We collected cardiac chamber quantification data using 2D echocardiography, which was performed according to American Society of Echocardiography guidelines. ${ }^{12)}$ Left ventricular end-diastolic dimension (LVEDD), left ventricular end-systolic dimension
(LVEDS), diastolic posterior left ventricular wall thickness (PWT), diastolic interventricular septum thickness (SWT), LVEF, and LV mass were assessed. The LV mass was calculated as follows:

$\mathrm{LV}$ mass $(\mathrm{g})=0.8 \times[1.04 \times\{(\mathrm{LVEDD}+\mathrm{PWT}+$ $\left.\left.\mathrm{SWT}^{3}-(\mathrm{LVEDD})^{3}\right\}\right]+0.6$.

Holter ECG analysis: Holter ECG was retrospectively analyzed by a medical technologist blinded to patient information, and the results were confirmed by a cardiologist. The analysis was performed using the SCM-8000 system (Fukuda Denshi, Tokyo, Japan). The following indices were analyzed hourly over a 24-hour period:

- Time domain analysis heart rate (HR), standard deviation of the NN interval (SDNN), proportion of NN50 (the number of pairs of successive NNs that differ by $>50 \mathrm{~ms}$ divided by the total number of NNs; pNN50).

- Frequency domain analysis low-frequency component (LF; 0.03-0.15 Hz), high-frequency component (HF; 0.15$0.4 \mathrm{~Hz}), \mathrm{LF} / \mathrm{HF}$ ratio.

To evaluate the circadian fluctuation of cardiac autonomic function, the 24 hour period was arbitrarily divided into day-time (8-22 o'clock) and night-time (22-8 o'clock).

Statistical analysis: The chi-square test, Student $t$ test, or 1-way analysis of variance was performed when appropriate. For post-hoc analysis, we adopted the Tukey-Kramer test to evaluate statistical differences among three groups depending on the medications. If the response variables were not normally distributed, we used a logarithmic transformation of the outcome variable to obtain normal distribution. Continuous data were shown as mean \pm SD for normally distributed data, and as median values [first quartile-third quartile] otherwise. A significance level of $5 \%$ was used for global test statistics.

Due to this cohort study's characteristics and heterogeneous patient population, we performed logistic regression analysis to identify independent factors contributing to the HRV parameters. Sexuality, age, comorbidities, he- 
Table II. Patient Characteristics according to Medications

\begin{tabular}{|c|c|c|c|c|c|c|c|c|}
\hline & \multicolumn{4}{|c|}{ Group-NKD } & \multicolumn{4}{|c|}{ Group-KD } \\
\hline & $\begin{array}{c}\text { No SLM } \\
n=304\end{array}$ & $\begin{array}{c}\text { BSB } \\
n=24\end{array}$ & $\begin{array}{c}\mathrm{ABB} \\
n=19\end{array}$ & $P$ value & $\begin{array}{c}\text { No SLM } \\
n=108\end{array}$ & $\begin{array}{c}\text { BSB } \\
n=26\end{array}$ & $\begin{array}{c}\mathrm{ABB} \\
n=15\end{array}$ & $P$ value \\
\hline Male & $122(40 \%)$ & $14(58 \%)$ & $14(74 \%)$ & 0.0050 & $59(55 \%)$ & $10(39 \%)$ & $11(73 \%)$ & 0.091 \\
\hline Age (years) & $57.9 \pm 15.5$ & $64.1 \pm 15.3$ & $62.9 \pm 12.3$ & 0.076 & $70.1 \pm 10.4$ & $71.5 \pm 12.3$ & $73.1 \pm 10.8$ & 0.53 \\
\hline Hypertension & $106(35 \%)$ & $16(67 \%)$ & $11(58 \%)$ & 0.0017 & $64(59 \%)$ & $22(85 \%)$ & $11(73 \%)$ & 0.040 \\
\hline sBP (mmHg) & $126 \pm 18$ & $124 \pm 18$ & $128 \pm 7.8$ & 0.77 & $126.0 \pm 18.6$ & $130.4 \pm 15.2$ & $124.7 \pm 21.1$ & 0.51 \\
\hline $\mathrm{dBP}(\mathrm{mmHg})$ & $75 \pm 12$ & $70 \pm 11$ & $77 \pm 8.6$ & 0.088 & $69.7 \pm 12.0$ & $71.5 \pm 8.2$ & $66.9 \pm 9.8$ & 0.47 \\
\hline Dyslipidemia & $82(27 \%)$ & $8(33 \%)$ & $9(47 \%)$ & 0.14 & $43(40 \%)$ & $9(35 \%)$ & $7(47 \%)$ & 0.75 \\
\hline Hyperuremia & $12(4 \%)$ & $1(4 \%)$ & $3(16 \%)$ & 0.058 & $33(31 \%)$ & $6(23 \%)$ & $5(33 \%)$ & 0.71 \\
\hline Ischemic heart disease & $17(6 \%)$ & $3(13 \%)$ & $3(16 \%)$ & 0.11 & $10(9 \%)$ & $8(31 \%)$ & $6(40 \%)$ & 0.0008 \\
\hline LVEF (\%) & $70 \pm 8$ & $70 \pm 8$ & $65 \pm 14$ & 0.044 & $70 \pm 9$ & $69 \pm 12$ & $65 \pm 13$ & 0.25 \\
\hline LV Mass (g) & $141.0 \pm 44.3$ & $138.0 \pm 41.1$ & $177.9 \pm 49.1$ & 0.0040 & $158.8 \pm 46.4$ & $168.0 \pm 56.5$ & $194.4 \pm 79.2$ & 0.065 \\
\hline Hemoglobin $(\mathrm{g} / \mathrm{dL})$ & $13.4 \pm 1.7$ & $13.4 \pm 1.1$ & $13.5 \pm 1.6$ & 0.98 & $12.5 \pm 2.1$ & $12.4 \pm 1.7$ & $12.8 \pm 1.5$ & 0.79 \\
\hline Serum creatinine $(\mathrm{mg} / \mathrm{dL})$ & $0.68 \pm 0.15$ & $0.74 \pm 0.12$ & $0.75 \pm 0.14$ & 0.047 & $1.81 \pm 2.00$ & $1.59 \pm 1.76$ & $1.32 \pm 0.46$ & 0.58 \\
\hline eGFR & $81.1 \pm 18.4$ & $74.9 \pm 12.4$ & $77.8 \pm 12.9$ & 0.20 & $40.3 \pm 15.8$ & $43.7 \pm 15.9$ & $42.7 \pm 11.8$ & 0.57 \\
\hline ACEi/ARB & $61(20 \%)$ & $9(38 \%)$ & $13(68 \%)$ & $<0.001$ & $52(48 \%)$ & $18(69 \%)$ & $10(67 \%)$ & 0.088 \\
\hline$\beta$-selective-blocking agent & $0(0 \%)$ & $24(100 \%)$ & $0(0 \%)$ & & $0(0 \%)$ & $26(100 \%)$ & $0(0 \%)$ & \\
\hline$\alpha \beta$-blocking agent & $0(0 \%)$ & $0(0 \%)$ & $19(100 \%)$ & & $0(0 \%)$ & $0(0 \%)$ & $15(100 \%)$ & \\
\hline $\mathrm{Ca}$ antagonist & $57(19 \%)$ & $7(29 \%)$ & $6(32 \%)$ & 0.21 & $50(46 \%)$ & $18(69 \%)$ & $9(60 \%)$ & 0.087 \\
\hline
\end{tabular}

In Group-NKD and Group-KD, patients' characteristics were compared between patients without sympatholytic medications (SLM), with betaselective-blockers (BSB), and with alpha-beta-blockers (ABB). P-value is based on the analysis of variance. See text for details.

moglobin value, echocardiographic parameters, and medications ( $\mathrm{ABB}, \mathrm{BSB}$, renin-angiotensin system inhibitors) were incorporated in the single variable regression analysis. For the multivariable regression analysis, we included the variables indicating statistical significance in the single variable regression analysis. Data analyses were performed using $\mathrm{JMP}^{\circledR}$ (SAS, Version 10, USA).

All authors had full access to the data, and have read and agreed to the manuscript as written.

\section{Results}

Patient population: Group-KD included older men with higher prevalence of hypertension, dyslipidemia, hyperuricemia, and diagnosed ischemic heart disease compared to Group-NKD. Although there was no significant difference in the mean LVEF, LV mass was significantly higher in Group-KD. Only 6 patients had reduced LVEF $<40 \%$ (4 in Group-KD, 2 in Group-NKD). Serum hemoglobin was significantly lower in Group-KD, and these patients took more medications as shown in Table I.

Table II shows the results of the subgroup comparison of patients' characteristics. In both Group-KD and Group-NKD, the patients who took $\beta$-blocking agents more frequently had hypertension. In Group-NKD, the patients under $\beta$-blocker therapy had larger LV-mass and were simultaneously taking renin-angiotensin inhibitors, and in Group-KD, tendency regarding these parameters were noted.

$\beta$-blockers: Fifty patients took BSB (Table II). Out of these 50 patients, bisoprolol, atenolol, metoprolol, and celiprolol were taken by $28,17,3$, and 2 patients, respectively. The daily dose of bisoprolol, atenolol, metoprolol, and celiprolol were $2.5-5 \mathrm{mg}, 12.5-50 \mathrm{mg}, 20-40 \mathrm{mg}$, and $200 \mathrm{mg}$, respectively. The only patients who did not take BSB drugs in the morning were those on atenolol $50 \mathrm{mg}$ $(n=1)$ and celiprolol with $200 \mathrm{mg}(n=2)$; they took their drugs twice daily (morning and evening). Thirty-four patients took 5-10 mg of carvedilol as an ABB every morning (Table II).

Heart rate parameters: The $\mathrm{HR}$ according to $\beta$-blocking agents are shown in Figure A. In both Group-KD and Group-NKD, the patients taking $\beta$-blocking agents had lower HR.

The frequency domain analysis results are shown in Figure B. In Group-KD, HF increased with the administration of $\beta$-blocking agents, particularly in patients taking BSBs, not only during the day-time but also during the night-time. In comparison, HF was increased by BSB only during the day-time in Group-NKD.

LFs according to $\beta$-blocking agents are shown in Figure C. Notably, $\beta$-blocking agents increased LF in Group-KD, although there were no differences in GroupNKD.

The analysis results on $\mathrm{LF} / \mathrm{HF}$ ratio are shown in Figure D. In Group-KD, the patients taking BSBs had a markedly lower $\mathrm{LF} / \mathrm{HF}$ ratio during the night-time. In Group-NKD, there was a decrease in $\mathrm{LF} / \mathrm{HF}$ ratio due to BSB use during the day-time but not during the nighttime.

\section{Regression analysis:}

HF (Table III) In Group-KD, BSB administration was independently related to higher HF throughout the day, and higher $\mathrm{Hb}$ values tended to be related with higher $\mathrm{HF}$. During the night-time, higher $\mathrm{Hb}$ values were significantly related to higher $\mathrm{HF}$. ABB did not significantly influence the HF throughout the day.

In Group-NKD, administration of both BSB and ABB significantly and independently increased HF, but only during the day-time. The age and the presence of hyperlipidemia negatively correlated to HF during the daytime. During the night-time, age and male gender were negatively correlated with HF.

There were no significant differences in HF between 

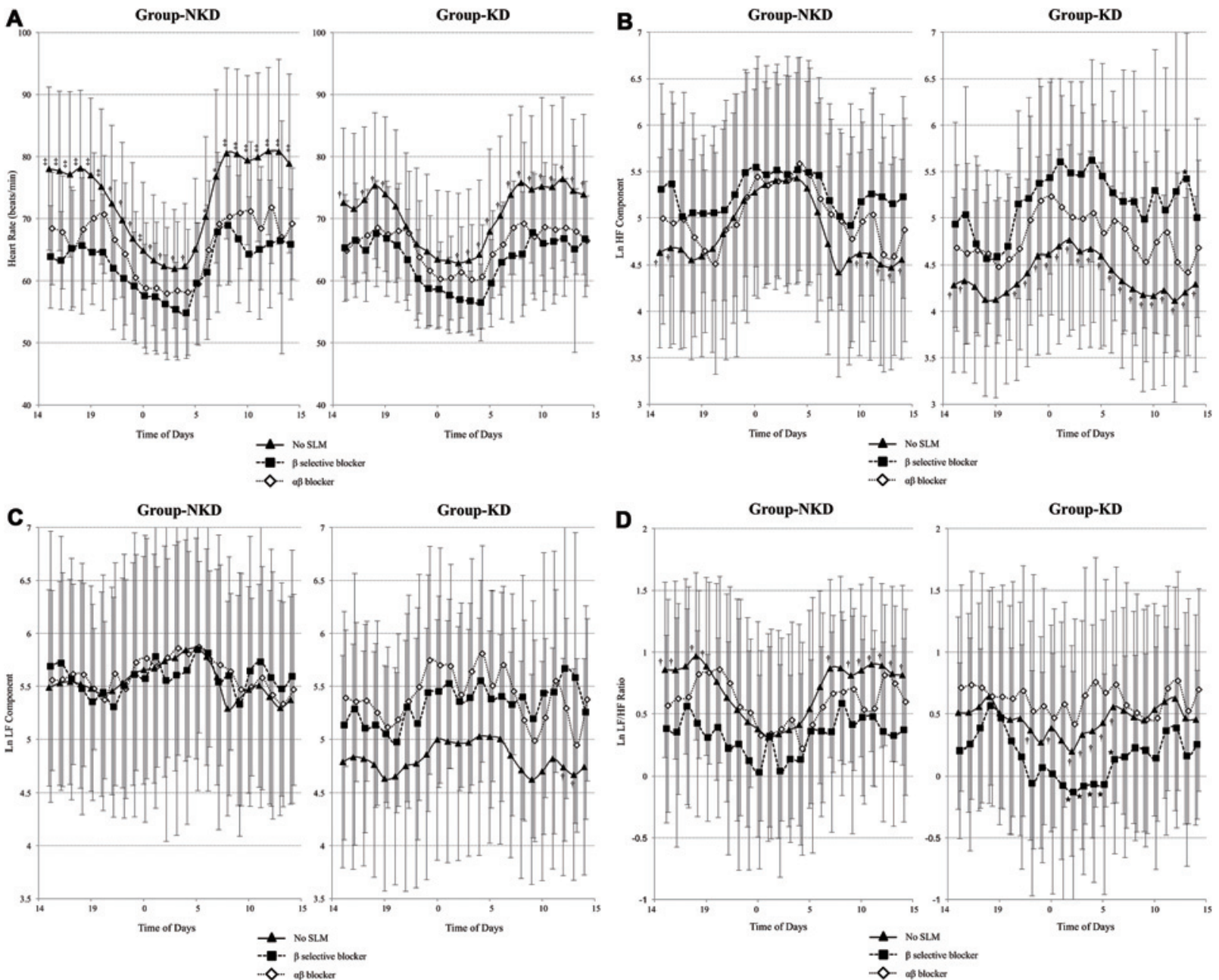

Figure. Circadian variation of heart rate (HR) parameters according to sympatholytic medications. A: Circadian variation of HR according to sympatholytic medications. Both sympatholytic medications (SLM), beta-selective blockers (BSB), and alpha-beta blockers (ABB) decreased HR during day-time and night-time. There were no significant differences between BSB and ABB. In Group-KD patients, although the baseline HR in the day-time was lower as compared to Group-NKD patients, HR under BSB or ABB treatment was on a comparable level. B: Circadian variation of high-frequency component according to SLM. In Group-NKD patients, higher HF during the day-time was noted with BSB treatment. In GroupKD patients, the baseline HF was lower as compared to the patients in Group-NKD and SLM increased HF throughout the day. C: Circadian variation of low frequency component according to SLM. In Group-NKD patients, SLM did not affect the LF. In Group-KD patients, LF was higher, but mostly without statistical significance, under SLM treatment. D: Circadian variation of low-to-high frequency ration according to SLM. In Group-NKD patients, BSB decreased the LF/HF ratio particularly during the day-time, but ABB did not obviously alter the LF/HF ratio. In GroupKD patients, BSB lowered the LH ratio during the night-time. ABB did not lower the LF/HF ratio. Moreover, ABB increased the LH ratio as compared to BSB during the night-time. ( $\$ P<0.05$, BSB versus no SLM and ABB versus no SLM; $\uparrow P<0.05$, BSB versus no SLM; $* P<0.05$, ABB versus no $\mathrm{SLM} ; \star^{\star} P<0.05, \mathrm{BSB}$ versus $\left.\mathrm{ABB}\right)$.

patients who were treated with BSB or ABB.

$L F$ (Table IV) In Group-KD, LF was significantly decreased in the older age group and lower $\mathrm{Hb}$ group throughout the day. BSB tended to be related with higher LF only during the day-time.

In Group-NKD, older age, the presence of HL and lower $\mathrm{Hb}$ correlated with lower $\mathrm{LF}$ throughout the day. The administration of BSB or ABB did not influence LF.

There were no significant differences in HF between patients who were treated with BSB or ABB.

LF/HF Ratio (Table V) In Group-KD, the LF/HF ratio during the day-time was significantly increased in the younger age group and male sex. BSB administration de- creased the $\mathrm{LF} / \mathrm{HF}$ ratio only during the night-time. $\mathrm{ABB}$ did not affect the LF/HF ratio both during the day-time or night-time.

In Group-NKD, BSB administration was associated with lower LF/HF ratio and the male gender, while higher $\mathrm{Hb}$ was associated with higher LF/HF ratio throughout the day. ABB administration was associated with lower LF/ $\mathrm{HF}$ ratio only during the night-time. The LF/HF ratio during the day-time was lower in the older age group.

Group-KD patients treated with $\mathrm{ABB}$ had a significantly higher LF/HF ratio than those who were treated with BSB, which was the only difference between treatment with $\mathrm{BSB}$ and $\mathrm{ABB}$. 
Table III. Regression Analysis Results (High Frequency)

\begin{tabular}{lccc}
\hline & Estimates & $95 \%$ CI & $P$ value \\
\hline HF in Group-NKD & & & \\
Day-time & & & 0.0003 \\
$\quad$ Age (1year) & -0.014 & $-0.021--0.0064$ & 0.0012 \\
$\beta$ selective blocker & 0.34 & $0.14-0.54$ & 0.0071 \\
$\alpha \beta$ blocker & 0.32 & $0.087-0.55$ & 0.047 \\
Hyperlipidemia & -0.13 & $-0.25--0.0019$ & 0.050 \\
LV mass (1g) & -0.0025 & $-0.0051--0.0000023$ & \\
Night-time & & & 0.0001 \\
$\quad$ Age & -0.021 & $-0.028--0.015$ & 0.023 \\
Male & -0.12 & $-0.22--0.016$ & \\
HF in Group-KD & & & 0.0049 \\
Day-time & & & 0.076 \\
$\beta$ selective blocker & 0.30 & $0.093-0.51$ & 0.0008 \\
Hb & 0.070 & $-0.0075-0.15$ & 0.023 \\
Night-time & & & $0.17-0.63$ \\
$\beta$ selective blocker & 0.40 & $0.014-0.19$ & \\
Hb & 0.10 & &
\end{tabular}

Logistic regression analyses were performed to test the relevance between clinical factors and heart rate variability. See text for details.

Table IV. Regression Analysis Results (Low Frequency)

\begin{tabular}{lccc}
\hline & Estimates & $95 \%$ CI & $P$ value \\
\hline LF in Group-NKD & & & \\
Day-time & & & \\
$\quad$ Age (1 year) & -0.024 & $-0.029--0.018$ & $<0.0001$ \\
Hyperlipidemia & -0.10 & $-0.20--0.0026$ & 0.044 \\
Hb & 0.061 & $0.0015-0.12$ & 0.045 \\
ACEi/ARB & -0.12 & $-0.24-0.0076$ & 0.066 \\
Night-time & & & \\
Age (1 year) & -0.021 & $-0.029--0.014$ & $<0.0001$ \\
Hyperlipidemia & -0.15 & $-0.27-0.024$ & 0.019 \\
Hb & 0.075 & $0.0050-0.15$ & 0.036 \\
LF in Group-KD & & & \\
Day-time & & & \\
Hb & 0.13 & $0.041-0.21$ & 0.004 \\
Age (1 year) & -0.016 & $-0.035-0.0014$ & 0.032 \\
$\quad \beta$ selective blocker & 0.19 & $-0.035-0.41$ & 0.098 \\
Night-time & & & \\
Hb & 0.14 & $0.056-0.23$ & 0.0014 \\
Age (1 year) & -0.019 & $-0.034--0.0048$ & 0.0095 \\
Male & 0.15 & $-0.012-0.32$ & 0.069 \\
\hline
\end{tabular}

Logistic regression analyses were performed to test the relevance between clinical factors and heart rate variability. See text for details.

\section{Discussion}

The major findings of this study were: 1) The influences of sympatholytic medication on cardiac autonomic function, as measured by HRV, were different depending on the presence of kidney dysfunction, 2) BSB administration was associated with higher parasympathetic HRV in patients with and without kidney dysfunction, 3) ABB was not associated with higher parasympathetic HRV, 4) BSB was associated with lower sympathovagal balance throughout the day in patients without kidney dysfunction, and only during the night-time in patients with kidney dysfunction, and 5) ABB did not affect sympathovagal balance in patients with kidney dysfunction.

To the best of our knowledge, this is the first detailed analysis of the effect of sympatholytic medication on patients with kidney dysfunction who do not have heart failure.

In patients with heart failure, sympathetic hyperactivity is related to worse prognosis, and sympathetic denervation improves cardiac function and clinical status. ${ }^{13)} \beta$ blockers are an established medication for improving patients' outcomes, and their use is not dependent on the presence of CKD. ${ }^{4,14)}$ In contrast, $\beta$-blockers are generally not first line treatments for hypertension in CKD patients $;{ }^{15)}$ rather, medications affecting the renin-angiotensin system are preferable because of their significant renalprotective effects. As a result, $\beta$-blockers are recommended for CKD patients as third-line therapy.

Previous studies linked augmented sympathetic activity in CKD patients to poor prognoses. ${ }^{5,16)}$ We have demonstrated that CKD presence can affect baseline circadian autonomic fluctuations. ${ }^{8)}$ The vagal function impairment was associated with $\mathrm{CKD}$, and the sympathovagal balance was affected by aging and hemoglobin value. We used these results to investigate the further deviation from these baseline circadian HRV fluctuations due to $\beta$-blocker administration.

In CKD patients, the nocturnal drop of sympathovagal balance is attenuated (Figure D). This may result renin-angiotensin system activation and possibly lead to sudden cardiac death in the early morning. ${ }^{17)}$ Theoretically, inhibiting sympathetic activity could improve CKD patient outcomes, similar to patients with heart failure. The dissociation between the hypothesis and current clinical practice suggests that autonomic function response to $\beta$ blockers may be different between CKD and non-CKD patients.

The efficacy of $\beta$-blockers, particularly atenolol, is inferior to other classes of antihypertensive drugs in patients with primary hypertension. ${ }^{18)}$ This inferiority can be attributed to the different characteristic effect on brachial systolic blood pressure and central systolic blood pressure. $^{19)}$ 
Table V. Regression Analysis Results (LF/HF Ratio)

\begin{tabular}{|c|c|c|c|}
\hline & Estimates & $95 \% \mathrm{CI}$ & $P$ value \\
\hline \multicolumn{4}{|c|}{ LF/HF ratio in Group-NKD } \\
\hline \multicolumn{4}{|c|}{ Day-time } \\
\hline Age (1 year) & -0.0087 & $-0.014--0.0035$ & 0.0011 \\
\hline Male & 0.11 & $0.023-0.20$ & 0.014 \\
\hline$\beta$ selective blocker & -0.16 & $-0.29--0.029$ & 0.017 \\
\hline $\mathrm{Hb}$ & 0.055 & $0.0073-0.10$ & 0.024 \\
\hline \multicolumn{4}{|l|}{ Night-time } \\
\hline Male & 0.20 & $0.12-0.28$ & $<0.0001$ \\
\hline $\mathrm{Hb}$ & 0.052 & $0.0057-0.098$ & 0.028 \\
\hline$\alpha \beta$ blocker & -0.16 & $-0.30--0.013$ & 0.033 \\
\hline$\beta$ selective blocker & -0.14 & $-0.27--0.0089$ & 0.036 \\
\hline Hypertension & -0.075 & $-0.15-0.0012$ & 0.054 \\
\hline \multicolumn{4}{|l|}{$\mathrm{LF} / \mathrm{HF}$ ratio in Group-KD } \\
\hline \multicolumn{4}{|l|}{ Day-time } \\
\hline Age (1 year) & -0.022 & $-0.032--0.012$ & $<0.0001$ \\
\hline Male & 0.13 & $0.022-0.24$ & 0.019 \\
\hline $\mathrm{Hb}$ & 0.047 & $-0.0082-0.10$ & 0.095 \\
\hline \multicolumn{4}{|l|}{ Night-time } \\
\hline Male & 0.21 & $0.098-0.32$ & 0.0003 \\
\hline Age (1 year) & -0.012 & $-0.023--0.0024$ & 0.015 \\
\hline$\beta$ selective blocker & -0.016 & $-0.30--0.015$ & 0.030 \\
\hline
\end{tabular}

Logistic regression analyses were performed to test the relevance between clinical factors and heart rate variability. See text for details.

However, several studies have demonstrated that the efficacy of vasodilating $\beta$-blockers, including carvedilol and nebivolol, is superior to conventional BSB without vasodilating properties. ${ }^{20-22)}$ Carvedilol has also been associated with a renal protective effect. ${ }^{23,24)}$

$\beta$-blockers and ABB decrease cardiovascular and allcause mortality in heart failure patients. ${ }^{25,26)}$ Renal dysfunction is a frequent comorbidity in patients with severe heart failure, and the concomitant treatment of reninangiotensin blockers and $\beta$-blockers is widespread. In the present study, severe heart failure patients were not included, as shown in Table II; therefore, the patient population differed from those in the aforementioned reports. In terms of CKD patients without left ventricular dysfunction, the advantage of $\beta$-blocker administration remains to be clarified. In the present study, administration of BSB, including atenolol, restored the nocturnal drop of LF/HF ratio. The prognostic influence of $\mathrm{BSB}$ in $\mathrm{CKD}$ patients who do not have left ventricular dysfunction should be further investigated.

We evaluated the effects of BSB and ABB (vasodilating) on cardiac autonomic function using HRV in the present study. There was no significant difference between $\mathrm{BSB}$ and $\mathrm{ABB}$ on $\mathrm{HR}$ in either Group-KD or GroupNKD.

In Group-NKD, BSB was associated with higher HF and lower $\mathrm{LF} / \mathrm{HF}$ ratio throughout the day, and $\mathrm{ABB}$ was associated with higher day-time $\mathrm{HF}$ and lower night-time $\mathrm{LF} / \mathrm{HF}$ ratio. This means that both $\mathrm{BSB}$ and $\mathrm{ABB}$ might augment parasympathetic activity and decrease sympathovagal balance.

In contrast, $\mathrm{BSB}$ and $\mathrm{ABB}$ had different effects on cardiac autonomic function in kidney dysfunction patients. BSB was associated with higher parasympathetic activity and lower sympathovagal balance in Group-KD; however,
$\mathrm{ABB}$ did not result in $\mathrm{HF}, \mathrm{LF}$, and $\mathrm{LF} / \mathrm{HF}$ ratio differences in this patient population.

Our present study data suggest that the operative mechanisms of $\beta$-blockers and ABB, especially on cardiac autonomic function, are different depending on the presence of kidney dysfunction in patients without severe heart failure.

Carvedilol has been reported to attenuate oxidative stress. ${ }^{27)}$ In CKD patients without heart failure, carvedilol may express antioxidative effects to the fore rather than its $\mathrm{ABB}$ effect. In the present study, HR and LF/HF ratio of CKD patients were significantly reduced by BSB but not by $\mathrm{ABB}$. Endothelial dysfunction and increased oxidative stress can be detected in early-stage CKD ${ }^{28)}$ Zepeda, et al. reported that carvedilol increased endothelial antioxidant capacity. ${ }^{27)}$ If the antioxidative effect of $\mathrm{ABB}$ can attenuate endothelial dysfunction in $\mathrm{CKD}$, then the relative vasodilation effect of $\mathrm{ABB}$ may explain the non-significant, night-time $\mathrm{HR}$ and $\mathrm{LF} / \mathrm{HF}$ ratio reductions in $\mathrm{CKD}$ patients under ABB therapy (Figures A, D). This hypothesis should be further investigated using animal studies along with prospective and controlled human studies. The clarification may lead to a different indication for carvedilol therapy in this patient population.

Moreover, a recent study indicates the central nervous system acts on autonomic systems via oxidative stress. ${ }^{29)}$ Further investigations regarding the effect on the central nervous system, which may be exacerbated by CKD, should be conducted.

Limitations: Due to a retrospective single center study, the selection and the dose of BSB and $\mathrm{ABB}$ were not unified in the cohort. An overall higher dose of $\mathrm{ABB}$ may increase $\mathrm{HF}$ activity and decrease the $\mathrm{LF} / \mathrm{HF}$ ratio further. Secondly, this is not a randomized study and not a paired design comparing same patients before and after $\beta$ - 
blockers use. The study design had two groups with different ages, which can affect autonomic function and the effect of sympatholytic agents (Table I). To overcome these limitations, we consecutively enrolled patients and incorporated clinical parameters in the multivariable model. As shown in Figure A, the HR in patients treated with $\mathrm{ABB}$ demonstrated a similar decrease to those treated with BSB. Therefore, we believe our data is reasonable in the clinical setting. However, further larger and randomized studies should be conducted to investigate the relevance of our data.

\section{Conclusion}

The effect of sympatholytic therapy depends on the presence of CKD and whether BSB or ABB is used. In CKD patients without severe heart failure, $\beta$-selective $\beta$ blockers could be associated with higher parasympathetic activity and lower sympathovagal balance.

\section{Disclosures}

Conflicts of interest: None.

\section{References}

1. Kaye DM, Lefkovits J, Jennings GL, Bergin P, Broughton A, Esler MD. Adverse consequences of high sympathetic nervous activity in the failing human heart. J Am Coll Cardiol 1995; 26: 1257-63.

2. Barretto AC, Santos AC, Munhoz R, et al. Increased muscle sympathetic nerve activity predicts mortality in heart failure patients. Int J Cardiol 2009; 135: 302-7.

3. Fonarow GC, Yancy CW, Hernandez AF, Peterson ED, Spertus JA, Heidenreich PA. Potential impact of optimal implementation of evidence-based heart failure therapies on mortality. Am Heart J 2011; 161: 1024-30.e3.

4. Ponikowski P, Voors AA, Anker SD, et al. 2016 ESC Guidelines for the diagnosis and treatment of acute and chronic heart failure: The Task Force for the diagnosis and treatment of acute and chronic heart failure of the European Society of Cardiology (ESC) Developed with the special contribution of the Heart Failure Association (HFA) of the ESC. Eur Heart J 2016; 37 2129-200.

5. Zoccali C, Mallamaci F, Parlongo S, et al. Plasma norepinephrine predicts survival and incident cardiovascular events in patients with end-stage renal disease. Circulation 2002; 105: 13549 .

6. Kidney Disease Outcomes Quality Initiative (K/DOQI). K/DOQI clinical practice guidelines on hypertension and antihypertensive agents in chronic kidney disease. Am J Kidney Dis 2004; 43: S1-290.

7. Bakris GL, Copley JB, Vicknair N, Sadler R, Leurgans S. Calcium channel blockers versus other antihypertensive therapies on progression of NIDDM associated nephropathy. Kidney Int 1996; 50: 1641-50.

8. Makimoto H, Fujiu K, Shimizu K, et al. Impaired heart rate variability in patients with non-diabetic chronic kidney disease prominent disruption of vagal control and daily fluctuation. IJC Metabolic \& Endocrine 2015; 9: 22-7.

9. Wali RK, Iyengar M, Beck GJ, et al. Efficacy and safety of carvedilol in treatment of heart failure with chronic kidney disease: a meta-analysis of randomized trials. Circ Heart Fail 2011; 4: $18-26$

10. Badve SV, Roberts MA, Hawley CM, et al. Effects of beta- adrenergic antagonists in patients with chronic kidney disease: a systematic review and meta-analysis. J Am Coll Cardiol 2011; 58: 1152-61.

11. Matsuo S, Imai E, Horio M, et al. Revised Equations for Estimated GFR From Serum creatinine in Japan. Am J Kidney Dis 2009; 53: 982-92.

12. Lang RM, Badano LP, Mor-Avi V, et al. Recommendations for cardiac chamber quantification by echocardiography in adults: an update from the American Society of Echocardiography and the European Association of Cardiovascular Imaging. J Am Soc Echocardiogr 2015; 28: 1-39.e14.

13. Geng J, Chen C, Zhou X, Qian W, Shan Q. Influence of renal sympathetic denervation in patients with early-stage heart failure versus late-stage heart failure. Int Heart J 2018; 59: 99-104.

14. Nitta D, Kinugawa K, Imamura T, Kato NP, Komuro I. High dose $\beta$ blocker therapy triggers additional reverse remodeling in patients with idiopathic non-ischemic cardiomyopathy. Int Heart J 2016; 57: 717-24.

15. Mancia G, Fagard R, Narkiewicz K, et al. 2013 ESH/ESC Guidelines for the management of arterial hypertension: the Task Force for the management of arterial hypertension of the European Society of Hypertension (ESH) and of the European Society of Cardiology (ESC). J Hypertens 2013; 31: 1281-357.

16. Johansson M, Elam M, Rundqvist B, et al. Increased sympathetic nerve activity in renovascular hypertension. Circulation 1999; 99: 2537-42.

17. Tislér A, Logan AG, Akócsi K, Tornóci L, Kiss I. Circadian variation of death in hemodialysis patients. Am J Kidney Dis 2008; 51: 53-61.

18. Lindholm LH, Carlberg B, Samuelsson O. Should beta blockers remain first choice in the treatment of primary hypertension? A meta-analysis. Lancet 2005; 366: 1545-53.

19. Manisty $\mathrm{CH}$, Hughes AD. Meta-analysis of the comparative effects of different classes of antihypertensive agents on brachial and central systolic blood pressure, and augmentation index. $\mathrm{Br}$ J Clin Pharmacol 2013; 75: 79-92.

20. Polónia J, Barbosa L, Silva JA, Bertoquini S. Different patterns of peripheral versus central blood pressure in hypertensive patients treated with $\beta$-blockers either with or without vasodilator properties or with angiotensin receptor blockers. Blood Press Monit 2010; 15: 235-9.

21. Kampus P, Serg M, Kals J, et al. Differential effects of nebivolol and metoprolol on central aortic pressure and left ventricular wall thickness. Hypertension 2011; 57: 1122-8.

22. Dhakam Z, Yasmin, McEniery CM, Burton T, Brown MJ, Wilkinson IB. A comparison of atenolol and nebivolol in isolated systolic hypertension. J Hypertens 2008; 26: 351-6.

23. Fassbinder W, Quarder O, Waltz A. Treatment with carvedilol is associated with a significant reduction in microalbuminuria: a multicentre randomised study. Int J Clin Pract 1999; 53: 51922.

24. Bakris GL, Fonseca V, Katholi RE, et al. Metabolic effects of carvedilol vs metoprolol in patients with type 2 diabetes mellitus and hypertension: a randomized controlled trial. JAMA 2004; 292: 2227-36.

25. Brophy JM, Joseph L, Rouleau JL. Beta-blockers in congestive heart failure. A Bayesian meta-analysis. Ann Intern Med 2001; 134: 550-60.

26. Packer M, Bristow MR, Cohn JN, et al. The effect of carvedilol on morbidity and mortality in patients with chronic heart failure. U.S. Carvedilol Heart Failure Study Group. N Engl J Med 1996; 334: 1349-55

27. Zepeda RJ, Castillo R, Rodrigo R, et al. Effect of carvedilol and nebivolol on oxidative stress-related parameters and endothelial function in patients with essential hypertension. Basic Clin Pharmacol Toxicol 2012; 111: 309-16.

28. Khaira A, Mahajan S, Kumar A, et al. Endothelial function and oxidative stress in chronic kidney disease of varying severity and the effect of acute hemodialysis. Ren Fail 2011; 33: 411-7.

29. Kishi T. Heart failure as a disruption of dynamic circulatory homeostasis mediated by the brain. Int Heart J 2016; 57: 145-9. 